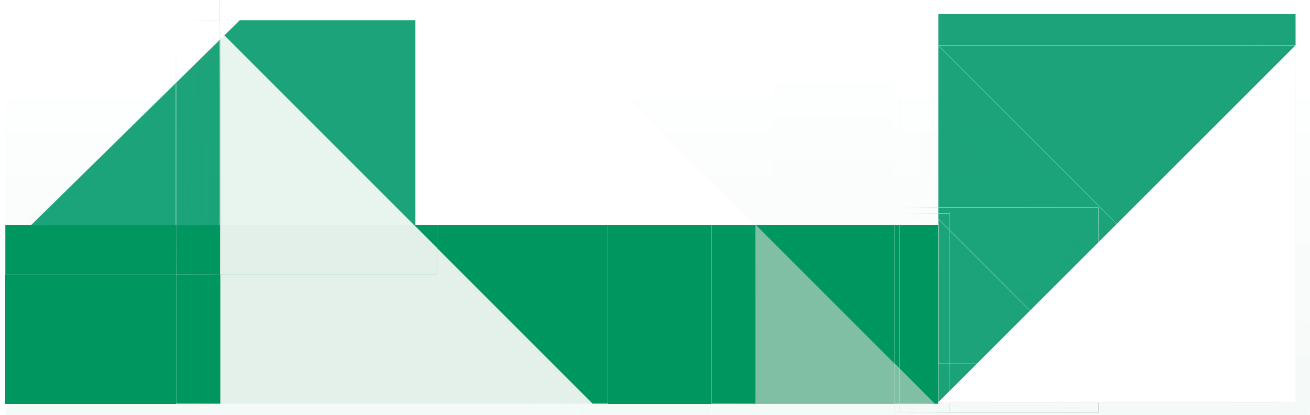

\title{
1) El concepto de tecnología escolar: una construcción de conocimiento profesional específico del profesorado de tecnología e informática
}

- The Concept of Technology at School: A Specific Professional Knowledge Creation by Technology and Computing Teachers

- O Conceito de Tecnologia de Escola: Um Conhecimento Profissional Específico, Construção de Professores e Informática

\section{Resumen}

En el presente artículo se plantea la recuperación de los sentidos y significados que el profesorado de tecnología e informática ha construido históricamente sobre el concepto de tecnología como categoría escolar de enseñanza. Esta investigación tiene como propósito principal identificar, caracterizar e interpretar el conocimiento profesional específico construido por los profesores de tecnología e informática sobre el concepto de tecnología escolar. El trabajo representa un estudio cualitativo de carácter interpretativo, que empleó como enfoque adecuado el estudio de caso múltiple. Contó con la participación de tres profesores licenciados en tecnología, expertos en la enseñanza del área: dos con más de 10 años de experiencia y uno con más de 30 años. Se realizaron en total 26 observaciones en registros de audio y video, empleando para ello un protocolo de observación. Igualmente se utilizó la técnica de estimulación del recuerdo, además de entrevistas semiestructuradas y lectura de los documentos institucionales relacionados con el área de tecnología e informática.

En cuanto al concepto de tecnología se PUEDE apreciar que es una creación histórica producto del mundo de la vida escolar, en la que el profesorado de tecnología e informática mantiene su aporte y participación especial. Este concepto debe comprenderse desde una perspectiva integradora del conocimiento, ya que la estructura de conocimiento del profesor es compleja y se alimenta de diferentes tipos de saber. Así, la tecnología escolar se entiende como: Una estructura relacional crítico-reflexiva que forma sujetos integrales, un dispositivo histórico de "razón polisémica del campo tecnológico", la unidad subjetiva y colectiva de creación y transformación del entorno, y un dispositivo de partici-

Mg. Jorge Mario Ortega Iglesias* Dr. Gerardo Andrés Perafán Echeverri**

\footnotetext{
Docente de planta de la Universidad del Magdalena. Estudiante del Doctorado Interinstitucional en Educación (DIE), Universidad Pedagógica Nacional de Colombia. Correo electrónico: joor212@gmail. COm. ORCID: orcid.org/0000-0002-6458-3650.

Docente de planta de la Universidad Pedagógica Nacional de Colombia. Director del grupo de investigación Por las Aulas Colombianas (Invaucol). Correo electrónico: anperafan@yahoo.com. orcid. org/0000-0002-9485-2997.
}

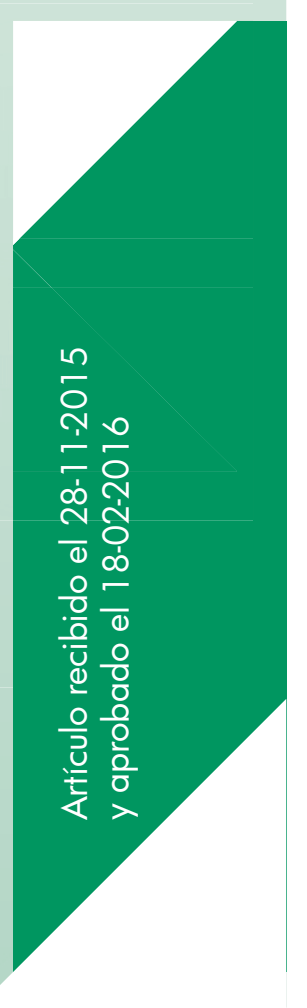


pación colectiva que afronta el hacer y saber hacer tecnológico en lo cotidiano. Esta comprensión del concepto de tecnología es la síntesis del sistema de ideas que integra el conocimiento específico del profesor, cuya integración obedece en principio al aula de clases como unidad representativa de significado, las fuentes de saber que nutren el conocimiento del profesor y sus respectivos estatutos epistemológicos, así como a un fenómeno discursivo de producción escolar de sentido.

Palabras clave

Tecnología escolar, conocimiento profesional específico, profesorado de tecnología

\title{
Resumo
}

Este artigo reflete sobre a recuperação dos sentidos e significados que os professores de tecnologia e informática têm construído historicamente sobre o conceito de tecnologia como uma categoria escolar de ensino. Esta pesquisa tem como objetivo principal identificar, caracterizar e interpretar o conhecimento profissional específico construído pelos professores de tecnologia e informática a propósito do conceito de tecnologia escolar. $\bigcirc$ trabalho representa um estudo qualitativo de caráter interpretativo, que utilizou como abordagem adequada o estudo de casos múltiplos, com a participação de três professores graduados de tecnologia de ensino e especialistas nesta área; dois deles com mais de 10 anos de experiência e um com mais de 30 anos. Um total de 26 observações, registradas em áudio e vídeos, foram realizadas, as quais empregaram um protocolo de observação. Da mesma forma foi utilizada a técnica de estimulação da memória, entrevistas semiestruturadas e leitura dos documentos institucionais relacionados à área da tecnologia e computação.

No que diz respeito ao conceito de tecnologia, se pode apreciar que é uma criação histórica do mundo da vida escolar, na qual os professores de tecnologia e informática reafirmam sua contribuição e participação especial. Este conceito deve ser entendido a partir de uma perspectiva integradora do conhecimento, uma vez que tal estrutura de conhecimento do professor é complexa e se alimenta de diferentes tipos de saber. Deste modo o conceito de tecnologia escolar é definido como: A estrutura relacional crítico-reflexiva que forma sujeitos integrais, um dispositivo histórico de "razão polissêmica do campo tecnológico", a unidade subjetiva e coletiva de criação e transformação do entorno, e um dispositivo de participação coletiva que explica o know-how tecnológico na vida cotidiana. Esta compreensão do conceito de tecnologia é a síntese do sistema de ideias que integra o conhecimento específico do professor, cuja integração se deve, em princípio, à sala de aula como uma unidade representativa de significado, às fontes de conhecimento que nutrem o conhecimento do professor e aos seus estatutos epistemológicos, bem como a produção escolar de sentido.

Palavras chave

Tecnologia escolar, Conhecimento profissional específico, Professores de tecnologia e de computação

\begin{abstract}
In this article the authors discuss the sense and meanings that ICT teachers have created over time on the concept of technology, seen as a school teaching category. The study aims at identifying, characterizing, and interpreting specific professional knowledge created by technology and computing teachers regarding the concept of technology at school. This is a qualitative research that interprets data taken from a multiple case study, which surveyed three highly-experienced technology and computing teachers (two of them with over 10 years' experience, and one with 30). Twenty-six observations were registered in audio and video following an observation guide. Other tools used were a stimulated recall methodology, semi-structured interviews, and the analysis of institutional documents related to the field.
\end{abstract}


Regarding the concept of technology, it can be seen that it is a historical construction, product of school life over time, which is, at the same time, an on-going process of participation and contribution of technology and computing teachers. This concept must be understood from a knowledge integration perspective in as much as it synthetizes multiple knowledge models. Thus, the concept of technology at school is identified with: (a) a critical-reflexive structure for the comprehensive education of the subject, (b) a historical dispositive of "polysemous reasoning in the field of technology", (c) the collective-subjective unit of milieu creation and transformation, and (d) a participation tool for the factual and procedural knowledge on a daily basis. This view of the concept of technology represents an integration of a system of ideas that make up the specific knowledge of teachers. Such integration conforms, beforehand, the classroom as a meaning representative unit; the epistemological principles and the sources of knowledge that support teachers' actions, as well as a discursive phenomenon of meaning production at school.

Keywords

Technology at school, specific professional knowledge, ICT teachers 


\section{Introducción}

Investigar sobre el conocimiento que históricamente ha construido el profesor desde sus prácticas de enseñanza como sujeto intelectual trabajador de la cultura es un referente de comprensión epistémica importante para resignificar la imagen y el papel de la profesión docente. Preguntarse por el conocimiento del profesor implica comprender un lugar de producción de sentido diferenciado que indaga por las características, las condiciones y la particularidad de saberes que construye el profesorado en el marco de su quehacer, cuyos saberes se consideran epistemológicamente distintos a aquellos que históricamente han tenido un lugar preponderante en la escuela.

En esta oportunidad nos hemos preguntado particularmente ¿̇Cuál es el conocimiento profesional docente específico construido por el profesorado de tecnología e informática asociado al concepto de tecnología? Nuestra intención principal es develar la red de sentidos y significados que atribuyen estos profesores durante sus prácticas de enseñanza, en función del concepto de tecnología desde el contexto escolar. Estos significados y sentidos atribuidos por los profesores de tecnología e informática al concepto de tecnología representan un conocimiento histórico creado por este profesorado bajo el principio intencional de enseñar, característica fundamental que hace posible la emergencia de sujetos aprendices de este concepto en la escuela.

Así, como consecuencia del desarrollo histórico del programa de investigación sobre el conocimiento profesional docente (Barinas, 2014; Barinas y Perafán, 2014; Bolívar, 2005; Bromme, 1988; Carr y Kemmis, 1988; Furió, 1994; García, 1997; Gil, 1991; Gimeno y Pérez, 1988; Hashweh, 2005; Jackson, 1968; Marcelo, 1993; Martínez, 2000; Park y Oliver, 2008; Perafán, 2004, 201 1, 2015; Ponte, 2012; Porlán y Rivero, 1998 y Shulman, 1986, 1987) el presente trabajo parte de la categoría conocimiento profesional docente específico asociado a categorías particulares planteada por Perafán (2011, 2012, 2013a, 2015), entendida como un referente teórico adecuado para comprender el fenómeno de producción de sentido en la escuela por parte del profesor.

La categoría conocimiento profesional docente específico es un sistema de ideas integrado que provoca la emergencia de conceptos o nociones particulares con los cuales trabaja el profesorado responsable de la enseñanza de las diferentes disciplinas escolares. Este sistema de ideas se compone de tipos de conocimientos que operan de manera integrada: saberes académicos, saberes basados en la experiencia, teorías implícitas y guiones y rutinas. Estos saberes no provienen de lugares foráneos, se originan a partir de estatutos epistemológicos fundantes (Perafán, 2004), entendidos como lugares de producción de sentido donde el profesorado de tecnología e informática mantiene una participación especial. Así, se entiende que los saberes académicos son producto de la transposición didáctica, los saberes basados en la experiencia se originan en la práctica profesional, las 
teorías implícitas provienen del campo cultural institucional y los guiones y rutinas se estructuran en la historia de vida.

El profesorado de tecnología e informática representa en este caso la comunidad de profesores en la que se ha pretendido identificar, caracterizar e interpretar un concepto de tecnología propio de su quehacer, que se foria en el marco de una red de sentidos diversos, fuentes de saber y demás elementos propios de la dinámica del aula de clases, y que particularmente obtiene legitimidad en el accionar discursivo cuando los maestros enseñan este concepto al estudiantado. La naturaleza del concepto de tecnología escolar como saber construido históricamente es parte de la subjetividad del profesor que otorga identidad al corpus de conocimiento con que educa, y se convierte en un aporte fundamental para la validez, el desarrollo y la vigencia de los saberes constitutivos de las disciplinas escolares, particularmente para el área de tecnología e informática.

\section{Metodología}

Esta investigación representa un estudio cualitativo de carácter interpretativo, en el que se empleó el estudio de caso múltiple $(\Theta \mathrm{A}, \Theta \mathrm{B}$, $\Theta C)^{2}$ (Creswell, 1998; Merriam 1998; Stake, 1995 y 2005; Wolcott, 1990 y Yin, 2003) como enfoque adecuado para su desarrollo (McCormick, 2006 y Middleton, 2008). En el estudio fueron seleccionados tres profesores licenciados en tecnología (que en adelante

La metodología específica que venimos trabajando en este tipo de investigaciones es un aspecto que se ha desarrollado de manera amplia y minuciosa y que se encuentra publicado en un capítulo completo del libro de G. A. Perafán (2015). Conocimiento profesional docente y prácticas pedagógicas. El profesorado como productor de conocimiento disciplinar-profesional. Bogotá: Aula de Humanidades. Nos permitimos invitar a quienes tengan interés en profundizar al respecto, a consultar el capítulo 2 del libro mencionado.

2 Denotación del caso múltiple (Stake, 1995, 2005). reconoceremos como Hefesto, Prometeo y Aracne): dos contaban con más de 10 años de experiencia en la enseñanza del área, y uno, con más de 30 años, ubicados en los niveles de educación básica primaria, secundaria y media, de dos instituciones educativas oficiales de Bogotá. Para este proceso de selección de maestros se tuvieron en cuenta los criterios de idoneidad, singularidad, complejidad y disponibilidad, que según Rodríguez, Gil y García (1999) atribuyen una condición crítica, única y reveladora para la comprensión de la realidad estudiada.

En este orden de ideas, se realizaron un total de 26 observaciones en registros de audio y video (una sesión semanal de 120 minutos aproximadamente, es decir de dos horas-clase por cada profesor) empleando un protocolo de observación diseñado para este proceso (Perafán, 201 1, 2015). Así mismo, cada docente participó de una serie de diálogos reflexivos a través de la aplicación de la técnica de estimulación del recuerdo (Clark y Peterson, 1990) y entrevistas semiestructuradas (Hernández, Fernández y Baptista, 2008; Janesick, 1998 y Perafán 2015). También se realizó una lectura minuciosa de documentos institucionales, como el PEI (proyecto educativo institucional), manuales de convivencia y documentos que definen los lineamientos para la enseñanza del área de tecnología e informática de ambas instituciones.

En cuanto al análisis de los datos se utilizó el dispositivo analytical scheme planteado por Perafán $(2011,2013 b, 2015)$, para quien este instrumento consiste en un constructo tanto teórico como técnico que reside en la conversión, a sendos tipos de argumentos, de 17 de las determinantes más significativas de la categoría Conocimiento profesional docente especifico asociado a categorías particulares, la cual a su vez integra cuatro tipos de saberes diferenciables. Se trata, entonces, de 
un dispositivo construido y validado específicamente para identificar, en la lógica y dinámica del orden discursivo de los profesores, el conocimiento profesional que mantienen, asociado a las categorías que enseñan. Los 17 tipos de argumentos ${ }^{3}$ así derivados, y asociados a la comprensión epistemológica de los cuatro saberes mencionados, constituyen la rejilla que se aplica al análisis de los diferentes episodios en los que se organizan los distintos datos. La información proveniente de cada uno de los instrumentos fue vertida a este dispositivo. Luego fue dividida y enumerada en 870 episodios, a cada uno de los cuales se le asoció por lo menos uno de los 17 tipos de argumentos mencionados para posteriormente realizar el proceso de triangulación de la información. Esto permitió la construcción de las categorías que definen el concepto de tecnología escolar, tal y como podrán verse reflejados en la sección de análisis e interpretación de los datos.

\section{Resultados}

El conocimiento específico del profesorado de tecnología e informática sobre el concepto de tecnología escolar como sistema de ideas integrado se conforma a partir de once categorías identificadas como figuras literarias, que explican la emergencia y el sentido que el profesor atribuye para enseñar el concepto de tecnología en la escuela; es decir, metáforas, ejemplos, símiles, imágenes, analogías, rituales, relatos y demás elaboraciones discursivas visibles y reiterativas en el aula de clases, principalmente en el dispositivo discursivo de los maestros para enseñar un concepto de tecnología específico a sus estudiantes.

Para una mejor lectura, primero se presentarán las once categorías de manera desagregada, posteriormente se mostrará cómo estas construcciones discursivas a manera de saberes se integran para dar origen al concepto de tecnología, qué tipos de relaciones guardan y cuál es su dinámica constitutiva.

\section{Los saberes académicos y su estatuto epistemológico fundante: La transposición didáctica asociada al concepto de tecnología escolar}

Estos saberes son el resultado de un entramado de relaciones complejas que circunscriben, entre otros, aspectos de carácter humano, contenido emocional, social, contextual e histórico. La naturaleza epistemológica que provoca la emergencia de estos saberes ocurre en el seno de los actos transpositivos de saber que realizan los profesores para enseñar el concepto de tecnología. Para ello, hemos de reconocer que la lectura de transposición didáctica ${ }^{4}$ que

3 El desarrollo de estos 17 tipos de argumentos puede verse en G. Perafán (2015, pp. 118 a 123).

4 Perafán $(2011,2013 a)$ partiendo de una reconceptualización de la noción de transposición didáctica -realizada desde la integración, al análisis de esta categoría, de algunos elementos sugeridos por Chevallard en el posfacio a la segunda edición del libro La transposición didáctica (1997)-, plantea que dicha 
abordamos, partiendo de los planteamientos de Perafán (2013a), da cuenta efectivamente del sentido que el profesor construye, que en este caso obedece a la intencionalidad de enseñar el concepto de tecnología como objeto de saber a un sujeto determinado en un contexto determinado. Esta dinámica de trabajo implica comprender que la noción de tecnología se construye en el marco de un proceso histórico y cultural de relación con el objeto de saber en juego por parte del profesor, en el cual se determinan las características distintivas del concepto de tecnología, que más allá de un proceso de modelación o reorganización del saber disciplinar, instala el sentido propio que el profesor ha construido.

Las siguientes son figuras literarias creadas por este profesorado en medio de los saberes académicos:

La metáfora del objeto como acción creadora, la cual contribuye a la construcción del sentido del concepto escolar de tecnología

La metáfora del objeto permite comprender este concepto como acción creadora a partir del trabajo y el hacer en la clase; es decir, de los diversos actos de creación del hombre y en particular de la participación que el estudiante tiene en la elaboración de diversos objetos tecnológicos. Esta metáfora implica identificar al estudiantado como un sujeto activo que participa en la creación de una variedad de productos tecnológicos y posibilita cambios en su realidad cercana. El objeto tecnológico se determina no como un elemento estático externo a la realidad del sujeto sino como parte constitutiva de la acción creadora de los estudiantes que introduce cambios en su

que da cuenta de una forma particular de producción de conocimiento y que es distinta del conocimiento cotidiano o conocimiento científico. mundo cercano y en su cultura. La tecnología constituye un acto o proceso de creación en diferentes dimensiones de lo humano por medio del cual se genera "cultura material". Veamos algunos episodios relacionados:

ӨA clase 1. 26-02-2013 (Hefesto)

\section{EP49}

Profesor: Miren, entonces vamos a hacer lo siguiente, miren, para entender un poquito ese concepto de tecnología. Por supuesto que esos primeros elementos que el hombre hizo hace 10.000 años y más atrás, que los utilizó como herramienta, ya eran productos tecnológicos, hasta ahí el hombre ya había desarrollado en algo la tecnología. Ahora, el computador, el celular, el televisor, todos esos elementos que ustedes conocen también hacen parte de la tecnología, pero esa es una tecnología digamos de punta, la última tecnología.

OA clase 4. 16-04-2013 (Hefesto)

\section{EP5}

Venimos haciendo, venimos desarrollando un proceso que nos debe permitir llegar a la realización de un objeto que las niñas van a regalar, żcierto? ¿̇Sí? Entonces las niñas estaban determinando como las posibles heee, 0 las posibilidades que tenemos para elaborar un objeto con ese fin. (Profesor revisa otro cuaderno de otra estudiante)

$$
\begin{aligned}
& \text { OB clase 3. 12-03-2013 (Prometeo) } \\
& \text { EP } 50
\end{aligned}
$$

Profesor: Bueno si usted me pregunta a mí qué es tecnología yo le podría decir a usted que es generar cultura material a través de elementos de uso cotidiano, eso es para mí la tecnología porque usted cada vez que fabrica algo está generando una cultura. Ya les expliqué la cuchara la otra vez żno? Ustedes vieron lo importante que fue la cuchara en la 
cultura, cómo un objeto tan simple, tan básico cambia la cultura eso es lo que vamos a hacer nosotros, cambiar culturalmente ¿̇sí?

La metáfora del relato de sucesos como transformación de la praxis del vivir humano, constitutiva de sentido parcial del concepto escolar de tecnología

Los relatos ${ }^{5}$ de sucesos antes que ser descripciones de hechos se constituyen en metáforas que portan sentidos escolares particulares sobre cambios tecnológicos visibles en el discurso de estos maestros. La movilización en forma de metáfora de estos hechos opera como una organización discursiva que orienta la enseñanza del concepto de tecnología de manera cercana y significativa, y se refiere principalmente a la tecnología como transformación de la praxis del vivir humano. Así, el concepto de tecnología se plantea como una serie de cambios y eventos que han transformado la vida del hombre en el marco del tipo de relación que este ha instaurado con diversos avances tecnológicos, y de los cuales los estudiantes, en su experiencia cotidiana, son testigos. Algunos episodios que evidencian en detalle esta metáfora se muestran a continuación:

OB clase 2. 05-03-2013 (Prometeo)

\section{EP158}

Profesor: Entonces llegaban las mamás algunas no sé si algunas de sus mamás o sus abuelas no creo que usted las haya visto pero de pronto le han contado que tenía que prender la estufa ya a gasolina que tenía que echarle una bomba, eso era una bomba allá en la casa, ahorita hoy en día usted llega tiene su microondas y la estufa a gas que se enciende [...] y fósforos; hoy en día. Pero anteriormente era otro proceso miren cómo ha evolucionado todo eso, entonces usted tenía que tener su estufa allá a gas donde era una bomba prácticamente que usted le echaba bomba con aire y le echaba combustible y lo que hacía era inyectar el combustible y prender un fogón, eso era lo que funcionaba anteriormente.

\section{EP159}

Profesor: Usted tenía que poner una estufa, una olla - por favor gracias de pronto esto le sirve algún día si se pierde en una Isla desierta-.

\section{Estudiantes: Jajajajaja}

Profesor: Que usted colocaba y ahí solo maíz pira. Entonces usted llega y colocaba la olla y usted tenía su aceite, tenía que saber más o menos cuál era la cantidad y eso solo lo hacía la experiencia, usted llegaba y le echaba poquito ya

5 El relato, soportado en el lenguaje articulado de carácter oral construido por el profesor, se entiende según Barthes et ál. (1972, p. 9) como un tipo de "sustancia" que ha estado presente "en todos los tiempos, en todos los lugares, en todas las sociedades: el relato comienza con la historia misma de la humanidad", que da vida, sentido e identidad, en este caso al trabajo del enseñante. 
sabía más o menos o algunas mamás tenían una tapita y sabían, son dos tapitas y ya sé que esa es la medida.

El ritual de las preguntas orientadoras como análisis, discusión, reflexión y organización mental para la solución de problemas en la vida del hombre, que favorece la construcción de saberes académicos asociados al concepto de tecnología

Las preguntas orientadoras identificadas corresponden a un proceso de interacción social, principalmente de interpelación al sujeto enseñante, que posibilita la emergencia del sentido asociado al concepto de tecnología escolar; es decir, en el orden discursivo que moviliza al profesorado de tecnología para enseñar la noción escolar de tecnología. Esta última se concibe también como interacción social cuyo fin es el análisis, la discusión, la reflexión y la organización mental para la solución de problemas en la vida del hombre. Dichas preguntas aparecen reiterativa y recursivamente hasta permitir la identificación, contextualización y comprensión respecto del sentido construido sobre el objeto de saber (concepto de tecnología) y, por supuesto, la emergencia del sujeto de saber (profesor y estudiante) en el marco de este tipo de interacciones. En este caso las preguntas orientadoras como ritual representan un trabajo intelectual de producción de conocimiento, que en el marco de continuos interrogantes origina un tipo de saber situado alrededor del concepto de tecnología, cuyo abordaje se da principalmente a la luz del análisis, la reflexión, la discusión y comprensión propia respecto de la resolución de diversos problemas que plantea el profesor durante la enseñanza interactiva.
Los saberes basados en la experiencia y su estatuto epistemológico fundante: la práctica profesional asociada al concepto de tecnología escolar

Estos saberes constituyen un cúmulo de conocimientos producto de la actividad docente, la cotidianidad en la escuela, la reflexión constante de la práctica, el intercambio de experiencias y diálogos en colectivos escolares y demás aspectos relacionados que enriquecen y logran orientar el accionar del profesor. Los profesores se desplazan y movilizan en medio de su actividad formadora, a partir de un tipo de racionalidad práctica que les permite actuar de manera oportuna e inmediata, de frente a la diversidad de situaciones que día a día los envuelven.

La práctica profesional que fundamenta esta racionalidad práctica, cuya dimensión es determinante para el desarrollo del ejercicio profesional del profesorado, representa también el lugar de creación de los saberes basados en la experiencia del profesor de tecnología e informática, y se constituye en una rica fuente de donde brotan saberes que se construyen y deconstruyen a partir de las vivencias que el profesional experimenta durante el día a día de su ejercicio, en el intercambio con el otro y en especial a partir de las condiciones y particularidades que le ofrece el escenario donde se desarrolla dicha práctica (Jackson, 1968). En este sentido, entendemos que la práctica profesional se instala como el estatuto epistemológico fundante de los saberes basados en la experiencia del profesorado de tecnología e informática asociado al concepto de tecnología, lugar donde se origina este tipo de saber constitutivo de la categoría conocimiento profesional específico del profesor. 
La metáfora del planteamiento de situaciones problema, necesidades y deseos del hombre como lugar vivencial, o experiencia cercana de interacción con los elementos del contexto, que favorece la construcción de saberes basados en la experiencia asociados al concepto escolar de tecnología

De manera recurrente y reiterativa estos profesores emplean en su discurso una serie de planteamientos expresados como situaciones problema, necesidades y en ocasiones deseos humanos, que orientan la enseñanza del concepto de tecnología. Este tipo de elaboraciones identificadas como metáfora en el discurso de los maestros encierran un sentido particular creado por el profesorado para enseñar a sus estudiantes este concepto, cuyo sentido tiene que ver con superar la comprensión de la tecnología como un sustrato teórico inmóvil y asumirla como un lugar vivencial, una experiencia cercana de interacción con los elementos del contexto que define y orienta el desarrollo creativo de la subjetividad en el aula, a partir del manejo de diferentes situaciones problemáticas de la cotidianidad visibles en estos planteamientos. Es decir, el concepto de tecnología se aborda implícitamente a partir del planteamiento y desarrollo de estas situaciones problema, necesidades y deseos de hacer en los educandos, que posibilitan su promoción como sujetos creativos y propositivos. Algunos episodios que reflejan esta comprensión son los siguientes:

OA clase 1. 26-02-2013 (Hefesto)

\section{EP73}

Profesor: A partir [...], bueno entonces vamos a mirar cómo se relaciona eso, cómo se lleva a cabo planear y proyectar, para eso tenemos que buscar cuáles son las necesidades que tenemos o el problema que queramos abarcar o cuál es el problema que queremos solucionar, partimos de ahí, de una necesidad o de un problema.

\section{EP74}

Profesor: Pero para poder establecer esa necesidad y ese problema, tenemos que mirar, tenemos que mirar nuestro contexto, porque la necesidad y el problema tiene que surgir es de lo que nosotros vivimos, porque recuerden que la tecnología nos permite solucionar son los problemas y los problemas nos lo presenta el contexto, o sea lo que estamos viviendo, lo que estamos desarrollando nosotros, lo que estamos viviendo, cuáles son los problemas que tenemos y qué podemos hacer para solucionar eso. Necesidad o problema. 
El ritual de proyectos y experiencias

exitosas como saber histórico

acumulado, producto de colectivos

escolares y promotor de la cultura

escolar, que posibilita la construcción

de saberes basados en la experiencia y

asociados al concepto de tecnología

El concepto de tecnología en este ritual se toma como un saber histórico acumulado que han producido colectivos escolares con la participación activa de profesores y estudiantes, cuyo saber aporta elementos significativos para la cultura en la escuela, lo que permite que, en medio de su participación, emerjan sujetos aprendices de este concepto. Así, la enseñanza de la tecnología se ve permeada por una variedad de prácticas y actividades en las cuales los estudiantes participan y reconocen la atribución de sentido que incorpora el profesor.

El concepto de tecnología se construye a partir de la inmersión y participación en actividades, ejercicios, proyectos y experiencias que los profesores reconocen como exitosas $y$ ponen en escena de manera reiterativa durante el desarrollo de sus clases. Un ejemplo claro de esta construcción de sentido se observa en los siguientes episodios:

\section{ӨC ENT 02-10-2013 (Aracne) \\ EP260}

Investigador: Bueno profe ¿̇Recuerda algún tipo de experiencia en su historia de vida que haya provocado un gran impacto para su comprensión de lo que es la tecnología?

Profesor: Los experimentos, yo diría que los experimentos hechos fuera del salón, eh dentro de ellos por ejemplo la bomba de ácido sulfúrico ¿̇sí? Entonces pues lo que necesitamos era que aquí el problema era que necesitábamos hacer una bomba. Algo que estallara y que nos generara un ruido que pudiera hacer y pues obviamente todo mundo lo manejaba por guerra.

\section{EP262}

Profesor: El de los cohetes de agua, pues es algo que después se promulgó en muchas partes que hagamos concursos de cohetes de agua que es simplemente presión de agua y a través de... de salir, la de los aviones żsí? Entonces aquí hicimos uno de aviones en donde el objetivo era caer en la mitad de la cancha del círculo, en la mitad de la cancha de fútbol pero lo tenían que enviar desde fuera y eso nosotros lo llamábamos el invento, nosotros decíamos el invento del mes. Y poníamos un reto, y nació de clase pero después ya se convirtió del colegio. Podía participar cualquiera de los que de los alumnos que estaban allí.

EP263

Profesor: Eh quiero hacer referencia que de ahí salieron lo de los carros que es que teníamos una feria, la feria que teníamos con otros colegios pero lastimosamente no teníamos la plata, no se dio por la parte otra vez administrativa, es que eso requiere muchos permisos.

La metáfora de los ejemplos como unidad de razón tangible, histórica y trascendente del objeto tecnológico, que favorece la construcción de sentido desde los saberes basados en la experiencia asociados al concepto de tecnología escolar

Esta metáfora se caracteriza principalmente por una comprensión de los ejemplos, que trascienden del hecho de ser comúnmente considerados como representaciones o instrumentos didácticos que aclaran e ilustran con mayor profundidad un contenido específico, 
a un proceso de determinación y caracterización del concepto de tecnología, que se expresa por medio del objeto tecnológico (constitutivo de razón y materialización) como una unidad de razón tangible, es decir, comprensible y palpable tanto en su dimensión abstracta como física, y además de carácter histórico y trascendente que provoca la emergencia de sujetos aprendices de este concepto. El cúmulo de experiencias creadas por el hombre y vistas como "objeto técnico" por parte del profesor encierran un conocimiento aprehensible, de carácter histórico y movilizador, que ha aportado importantes elementos al desarrollo de la humanidad y que en esta oportunidad educa sujetos en el aula de clases.

La identidad que la metáfora del ejemplo otorga al concepto de tecnología escolar en el aula evidencia una variedad de productos tecnológicos elaborados por el hombre, que en su interior contienen ricas formas de conocimiento histórico que han representado valiosos aportes para el desarrollo de nuestra cultura, ya que trasciende la comprensión del objeto tecnológico como algo acabado, hacia su interpretación como un mundo de posibilidades de construcción de conocimiento, gracias a la naturaleza de razón tangible con que se aborda.

El siguiente episodio recoge el sentido que constituye la metáfora del ejemplo.

OB ENT 23-09-2013 (Hefesto)

\section{EP34}

¿Cuáles estrategias utiliza para enseñar la noción de tecnología?

Profesor: Eh bueno, una es esa parte donde yo indago sobre esa concepción que pueda tener el estudiante de tecnología para poder hacer que esa noción que ellos tienen también evolucione, entonces por ejemplo a veces yo pregunto ¿qué entienden por tecnología? ○ ¿Cómo podríamos ejemplarizar la tecnología? Entonces ellos empiezan a nombrar elementos que se han producido a partir de la tecnología entonces hablan del televisor, hablan del celular y entonces yo trato de mostrarles que ese es un producto de la tecnología y se ha desarrollado a través de la historia y que tiene mucha historia y mucho conocimiento y que si el conocimiento no se hubiera desarrollado ese producto tecnológico tampoco habría llegado a materializarse.

Las teorías implícitas y el campo cultural institucional como su estatuto epistemológico fundante asociado al concepto de tecnología

Identificar las teorías implícitas que orientan y soportan el discurso del profesor se convierte en una tarea compleja, ya que intentamos comprender las relaciones existentes entre el discurso que se hace explícito durante la enseñanza y las teorías que subyacen y soportan este discurso, aunque el sujeto profesor no sea capaz de verbalizarlas, es decir, no pueda, de manera consciente, darse cuenta de las teorías que dirigen su accionar discursivo. 
Para el caso de las teorías implícitas asociadas a la noción de tecnología escolar, reconocemos que existe un lugar de producción de sentido que permea de manera tácita el discurso del profesor. Dicho lugar permite la vida, la reproducción y el trabajo de los saberes en la escuela, pero más desde una postura organizativa que establece pautas y propósitos alrededor de la enseñanza de los saberes escolares; nos referimos al campo cultural institucional, que se traduce en la puesta en escena de los referentes teóricos institucionales, como el modelo pedagógico, el plan de estudios del área de tecnología e informática, el sistema de evaluación, proyectos transversales, libros de textos, entre otros documentos que posibilitan la formación del sujeto. De esta manera el campo cultural institucional comprende el estatuto epistemológico fundante de las teorías implícitas que habitan en el discurso del profesor y que se vinculan con el concepto de tecnología que mantienen los profesores de tecnología e informática durante su enseñanza. A continuación se presentan las figuras literarias asociadas a este tipo de saber:

La metáfora de la formación humana como tecno-ética asociada a la recuperación del sujeto hombre instalado en una suerte de determinismo tecnológico, en la construcción parcial del sentido del concepto de tecnología escolar

Una de las teorías implícitas identificadas parte del reconocimiento de una metáfora recurrente en el discurso de los profesores, de la cual no son conscientes a plenitud. Nos referimos a una serie de argumentos relativos a la formación humana visibles en su dispositivo discursivo, que permean la enseñanza del concepto de tecnología, entendida como tecno-ética asociada a la recuperación del sujeto hombre instalado en una especie de determinismo tecnológico que orienta sus modos de vivir y de actuar en diferentes contextos.

La metáfora implícita de la formación humana como técno-ética implica que los estudiantes comprendan la recuperación del sujeto hombre, muchas veces absorbido por la masificación de dispositivos tecnológicos que determinan nuestros modos de ver, sentir y actuar en el mundo que nos rodea. El sentido que le atribuye este profesorado destaca aspectos constitutivos de la integridad del sujeto, con referencia al rescate de valores humanos como la responsabilidad, el amor, la sinceridad, la solidaridad, el compartir con el otro y de manera particular la recuperación del núcleo familiar, es decir, el símbolo de unidad que debe representar la familia en los estudiantes; ello, como la acepción positiva que prima sobre la desaparición del sujeto hombre-humano en un mundo tecnológico.

Los siguientes episodios ofrecen una evidencia del papel que representa la formación humana como teoría implícita durante la enseñanza.

OA Clase 5. 30-04-2013 (Hefesto)

\section{EP32}

Profesor: A ver allá, lo que yo pretendo es que veamos la película, que veamos cuál es la crítica que se hace en relación al desarrollo de la tecnología pero que también podamos hacer un paralelo con lo que nos pasa hoy. La película tiene unas escenas incluso donde muestra que literalmente y físicamente las máquinas consumen al hombre casi que se lo traga, ibueno allá lo van a ver! Es solamente un adelanto.

EP33

Profesor: Entonces qué tiene que ver con lo que nos pasa hoy en día, ¿̇Qué tiene que ver esto con lo que nos pasa hoy en día? Pues 
por ejemplo: ¿Qué tiene que ver eso cuando en la casa hay dos y tres televisores y cada individuo está por allá?, pero bueno uno lo ve, o bueno hay un televisor, un computador, un Play Station y cada uno por allá metido manejando un aparato de esos y ¿̇Qué pasa con las relaciones humanas, con las relaciones con el papá, con la mamá, con el hermano? ¿̇Cómo se tornan? No se tornan porque solamente está [...] digamos llega y no le dice [...] no se comunican con el papá sino se van y se conectan a internet [...]

\section{EP34}

Profesor: Bueno, entonces eso es lo que tenemos, lo que yo quiero que ustedes vean y para qué, para qué vamos a ver eso, para que nosotros entremos en conciencia y empecemos a darle desde nosotros mismo un buen uso a los productos tecnológicos, ¿̇listo?

La metáfora de la protección y cuidado del medioambiente como bio-técnica que aporta un sentido parcial al concepto de tecnología escolar

El discurso de los profesores también se encuentra permeado por una serie de orientaciones que, a manera de metáfora, dan cuenta sobre la importancia que tiene el cuidado del medioambiente para la salud y vida de las personas, lo que nombramos como bio-técnica. Estas orientaciones aparecen de forma reiterativa durante las clases de tecnología a la hora de realizar determinadas prácticas y ejercicios que posibilitan la enseñanza del concepto de tecnología escolar, lo que indudablemente conduce a su integración como componente del sentido general que connota este concepto escolar. Las orientaciones sobre la promoción y protección del cuidado del medioambiente brindan elementos que se articulan directamente al sentido que el profesor pone en juego cuando enseña el concepto de tecnología en el aula, es decir, la tecnología supone también una serie de prácticas que promueven la formación de un sujeto responsable en el cuidado y la protección del entorno escolar y, por supuesto, del medioambiente.

\section{Guiones y rutinas y su estatuto epistemológico fundante: la historia de vida asociada al concepto escolar de tecnología}

El hacer de la práctica docente conjuntamente con el discurso de los profesores de tecnología e informática en esta investigación se encuentra permeado por una serie de guiones y rutinas que, por definición (cf. Perafán 2011, 2015) otorgan identidad epistemológica a la enseñanza del concepto de tecnología escolar. En otros términos, existe una organización distintiva de saber y hacer en el aula, que de manera tácita dirige el curso de la clase para enseñar este concepto. Así, los guiones y rutinas se definen en este espacio como un conjunto de esquemas de actuación de carácter implícito que posibilitan la predicción y el control del curso de diferentes acontecimientos, particularmente de los esquemas de actuación es- 
colar por parte de estos profesores en función de la enseñanza del concepto de tecnología.

La característica principal de los guiones y rutinas identificados en estos maestros tiene que ver con el lugar de origen de tales esquemas, es decir, la historia de vida personal como el estatuto epistemológico fundante (Perafán 201 1 , 2015). En otras palabras, la historia de vida de los profesores se constituye como un sustrato intelectual experiencial tácito de donde en primer lugar recuperan su historia como individuos pertenecientes a una cultura y a una comunidad de sujetos, hecho que aporta elementos importantes a la configuración de su subjetividad. Y, en segundo lugar, esta subjetividad inseparable de su condición de sujeto enseñante se instala a su vez como dispositivo estructurante de sentido en el aula de clases; de este modo origina una rica y variada forma de conocimiento denominado guiones y rutinas de acción, asociado al concepto de tecnología escolar.

Como consecuencia de este devenir histórico en la configuración de la subjetividad profesoral que resalta aspectos personales, también se plantea que esta estructura generatriz de sentido ha definido tanto de manera tácita como reprimida formas propias de enseñanza que circulan en el aula de clases. A continuación se presentan en detalle las figuras discursivas que hemos identificado en estos profesores.

El guion de la pregunta ¿̇qué es? como saber fundado en un tipo razón técnica constitutiva del sujeto, asociada al descubrir, crear y proponer, que aporta implícitamente a la construcción del sentido general del concepto de tecnología escolar

En una primera impresión, la pregunta por el qué es podría considerarse como un elemento orientador que le permite al sujeto que plantea el cuestionamiento indagar por una definición precisa de lo que pregunta. Es como si, de manera mecánica e inmediata, preguntar por el qué es nos situara en una definición instantánea sin mayor alcance. No obstante, el uso reiterativo e insistente de la pregunta ¿̇qué es? evidenciado en los registros de observación de clase del caso múltiple estudiado nos hace comprender un direccionamiento diferente que el profesor emplea cuando plantea la pregunta de manera reiterativa e insistente: este obedece a la instalación de un espacio de reconocimiento y organización colectiva de ideas que aportan sentido a la estructuración de la temática específica que se quiere abordar. El sentido atribuido define el concepto de tecnología como una forma de conocimiento que emerge de manera colectiva entre profesores y estudiantes, producto de la duda sistemática que provoca la emergencia de un tipo de razón técnica constituyente del sujeto, la cual se relaciona con el descubrir, crear y proponer implicado en las actividades propias de la enseñanza de la tecnología. Veamos algunos ejemplos de esta estructura de sentido:

\section{OA Clase 1. 26-02-2013 (Hefesto) \\ EP37}

[Profesor se para enfrente del tablero y lo señala.] Bueno, entonces vamos a mirar qué es la tecnología, vamos a mirar qué es la tecnología.

\section{EP39}

Profesor: Entonces aquí las niñas que me quieran ayudar, levantan, levantan la mano para tratar de hacer una definición o mirar qué es eso de tecnología.

Estudiante: La tecnología es algo de la evolución. 
EP40

Profesor: Bueno, tú, a ver, oye... estoy haciendo una pregunta. ¿̇Para ustedes, qué entienden por tecnología? A ver ¿̇Tú qué entiendes por tecnología? A ver. ¿No? A ver, Otra niña que me ayude. ¿̇ué será eso de la tecnología? A ver, a ver. ¿̇Ninguna?, ya les dije, ya les dijimos.

Estudiante: Algo parecido a informática.

\section{EP4 1}

Profesor: Algo parecido a informática. Oye, ¿̇Qué entiendes por tecnología? O ¿̇sa palabra la habías escuchado antes, no la habías escuchado? ¿Qué entiendes por eso? En voz alta para que te escuchemos.

Estudiante: Tecnología es como la informática, sirve para los trabajos, hacer origami. [No se escucha claro]

\section{El guion del reto como desequilibrio que se integra a la condición} del saber y el saber hacer del sujeto que lo incorpora, como sentido particular asociado al concepto de tecnología escolar

El reto instituye en el aula de clase una constante de apelación a la razón del sujeto estudiante, que no es solo determinar situaciones de conflicto cognitivo; es decir, ejercicios en la clase que enfrentan y problematizan el hacer del estudiante en el aula, hacia la incorporación de un espacio particular de caracterización y construcción del concepto de tecnología al que acuden los maestros. Este tipo de guion que posibilita la emergencia del sentido durante las clases se define entonces como una constante implícita de apelación a la razón creadora en los educandos, que permite la construcción propia del concepto de tecnología, que supone un tipo de desequilibrio entre lo que sabe y puede hacer el estudiante, en el cual se tiene en cuenta el dominio creativo de los elementos cercanos que tiene a su disposición para enfrentar diversas situaciones de su cotidianidad. De esta manera, los retos se convierten en un tipo de detonante de saber que permite un abordaje propio sobre la enseñanza de este concepto. Veamos a continuación algunos ejemplos de las situaciones de la clase de tecnología que caracterizan los retos:

ӨC Clase 1. 06-03-2013 (Aracne)

P7

Profesor: Hoy vamos a intentar entrar al juego y primero lo vamos a conocer y después de conocerles voy a colocar retos de construcción, de acuerdo, a ver si sí somos inventores, entonces vamos a comenzar a desarrollar las actividades. Necesito que lleguen por lo menos hasta el nivel cinco y yo voy pasando por cada uno de los computadores. [sic] 
EP9

Estudiante: [...]

Profesor: Inicio, todos los programas, tecnología, listo muy bien. Listo, comencemos. Bien, ¿̇tú ya vas en ese nivel verdad?

EP143

Profesor. Ah bueno, listo muy bien, vamos a hacer el segundo reto. Segundo reto, el segundo reto es que vamos a colocar en la parte superior vamos a colocar una bola, la que ustedes deseen, sea de boliche sea de tenis, si usted le da "start" se va a dar cuenta [de] que él [sic] se demora entre 1 y 2 segundos en llegar a la parte de abajo de la pantalla. Yo necesito que se demore en recorrer la pantalla como mínimo 20 segundos porque si usted le da "start", iplop! un segundo y se fue de la pantalla. Yo necesito es que se quede en la pantalla 20 segundos.

EP152

[Revisa el trabajo de los estudiantes por computador)]

Profesor: Ese es el siguiente el siguiente reto pero va muy bien, o sea se me adelantó en un reto, pero ese es el siguiente reto. $\mathrm{Si}$ quieres síguelo trabajando, el siguiente reto es eso que esa bolita siempre este dentro de la pantalla, siempre este dentro de la pantalla, ya, ya, ya dale...
El guion de las frases asociadas a la dominación y dependencia tecnológica como crítica respecto al componente de doblegación de la voluntad, individual y colectiva, que supone la integración acrítica de la tecnología contemporánea a la vida cotidiana de los sujetos, sentido que se integra a la construcción del concepto de tecnología escolar

En el dispositivo discursivo de los profesores de tecnología e informática que participan en esta investigación aparecen con frecuencia una serie de frases y palabras encaminadas a identificar un tipo de dominación y dependencia tecnológica a la cual constantemente nos vemos enfrentados.

Ese proceso de imposibilidad de escape y adaptación rápida al uso del andamiaje tecnológico supone además la instalación de un dispositivo de represión cultural que al mismo tiempo que invita al sujeto profesor para el reconocimiento apresurado y la utilización de determinados dispositivos tecnológicos en la escuela, opera como una estructura que guarda en el inconsciente colectivo de los profesores deseos y sentimientos propios asociados a formas de aceptación o rechazo de tal dispositivo cultural. Nos referimos en este sentido a las formas en que estos profesores enseñan tecnología en la escuela, en particular sobre la comprensión del mismo concepto de tecnología. Así pues, históricamente asumimos que el dispositivo discursivo de los profesores que enseñan tecnología e informática se ve permeado por un tipo de estructura inconsciente que soporta el devenir de la clase de tecnología e informática y aporta elementos importantes para la construcción de un sentido crítico y liberador asociado a la noción de tecnología escolar. A la luz de estos argumen- 
tos se reconoce que el concepto de tecnología es portador de una perspectiva crítica y reflexiva frente el dispositivo tecnológico que se ha "naturalizado" en la vida del hombre contemporáneo, cuyo sujeto muchas veces es "sometido y doblegado" a una forma de culturización propia del andamiaje tecnológico, en el cual se reconoce también su condición acrítica alrededor de la instalación de este dispositivo.

Por ejemplo, el verbo someter aparece en diferentes expresiones que los profesores utilizan a lo largo de su discurso: "sometido por esa situación", "después de haber sido sometido", "que sometían al hombre y nos somete". Estas frases desempeñan un papel determinante en función de la enseñanza del concepto de tecnología, ya que su recurrente aparición, más allá de vincularse con una estrategia particular de enseñanza, orienta el sentido que los maestros inconscientemente aportan respecto de la importancia de la tecnología en la vida de los estudiantes; es decir, de una condición de sometimiento por parte de algunos dispositivos culturales de la cual debemos ser sujetos críticos y proactivos.

ӨA Clase 7. 14-05-2013 (Hefesto)

\section{EP38}

Profesor: Esa película como ustedes la han visto es una película que tiene una crítica frente a lo que es el desarrollo de la modernidad, de la tecnología y en ella nos muestra injusticias.

Estudiante ¿̇Cómo así?

Profesor: Muestra injusticias, ahí se dan unas injusticias de tipo, a ver, a ver - oye niña ya por favor- nos muestran unas situaciones que son críticas y que son de alguna forma generadas por el desarrollo de esa tecnología; es una tecnología donde el ser humano de alguna forma es sometido por esa situación, a ver, las niñas ahí, ya.

Ya, ¿̇se acomodó?

P43

Profesor: Entonces, ahí se ve un reflejo de lo que era la tecnología en ese momento. Las máquinas żcómo eran las máquinas? Eran máquinas digamos para producciones, eeeh para altas producciones que ya se empezaban a ver desde ese entonces y que sometían al hombre a unas rutinas que lo llevaban, lo llevaban hasta la enfermedad como pasó ahí con el protagonista żno? Que termina en, en un hospital después termina en la cárcel por injusticias de tipo social.

EP46

Profesor: Por ejemplo, recordemos esa escena donde, donde la, donde...el protagonista después de haber sido sometido como a un ritmo eeeh, acelerado de trabajo en una, en una rutina que lo enferma hasta llevarlo al, al borde de 
la locura, él termina siendo comido por una máquina, ¿̇se acuerdan? Que la máquina literalmente se lo come...

\section{EP48}

Estudiante: Nos enferma también. (Lluvia de ideas)

Profesor: A ver, miren el computador también nos enferma ¿Cómo nos enferma el computador?

Estudiantes: Cuando todo el tiempo ahí...o no tener gafas y a unos los enferma de los ojos.

Profesor: Lo enferma de los ojos, nos somete ża qué? A estar todo el tiempo pegado al computador se vuelve adictivo se nos olvidan que alrededor tenemos otros seres humanos que nos acompañan.

Ahora bien, cuando observamos con atención que el someter o sometimiento ha sido una de las experiencias traumáticas que ha tenido el profesor en su historia de vida personal, y que más allá de ser una frase o palabra reconocida a nivel discursivo se asocia a una condición como sujeto del mundo de la vida (desplazado por la violencia) que se juega en medio de deseos de superación y las frustrantes condiciones de vida que definieron en parte su proceso de formación educativa, entendemos que el someter o sometimiento tiene que ver con un esquema de actuación de carácter inconsciente que aparece en su papel como sujeto enseñante a manera de respuesta ante el hecho de ser sometido, es decir, ante la subordinación, sumisión a la voluntad de otro. Por consiguiente, estas frases no solo representan una condición que explica una posible comprensión de la tecnología a partir de la relación que mantienen los sujetos con la apresurada incorporación de dispositivos tecnológicos en su entorno, sino además un guion de carácter inconsciente construido en la historia de vida personal que define algunos elementos críticos se relacionados con el sentido que los maestros han construido y que su estructura inconsciente pone en juego durante la enseñanza a través de estas frases. Este sentido se ve representado en la capacidad subjetiva de respuesta frente a situaciones que doblegan nuestra voluntad de actuar.

Otra de las frases asociadas que se encuadran en este guion tiene que ver con el ser esclavo de la tecnología. En los siguientes episodios de los profesores Hefesto y Prometeo, correspondientes a los registros de observación y las entrevistas, se puede apreciar la utilización de frases y palabras relacionadas con el ser esclavo de las tecnologías.

OA ENT. 23-09-2013 (Hefesto)

\section{EP68}

Profesor: - lba en un bus yo- esas son las cosas que te permite reflexionar y en entonces dos papás en un celular clavado ahí con un niño acá y pumm el niño dio el bote y se pega su porrazo y casi que ni se dan cuenta. Ahí estamos viendo cómo, a mí me parece, cómo la tecnología deshumaniza y es eso, es como ese tipo la ambición que genera en otros seres humanos para someter y esclavizar al otro creo que ahí por eso hablo de que deshumaniza también.

Caso OB Clase 1. 26-02-2013 (Prometeo)

\section{EP175}

Profesor. Cuando usted está trabajando y que su jefe le diga, le mandé un correo a las 5 de la mañana y usted no lo leyó, usted se está volviendo esclavo de la tecnología, ojo con eso, usted no puede vivir pendiente de su correo electrónico, hace algunos años no existían y no todos andábamos pendientes porque no todos tenían celular con plan de datos, y la gente era más feliz. 


\section{EP176}

Profesor: Entonces pilas con eso, usted tiene aquí [que] aprender a utilizar la tecnología adecuadamente, no se vuelva esclavo de ella. Muchos de ustedes yo los he visto con los celulares ahí caminando y ni siquiera miran al frente, yo les dije la vez pasada, ustedes ya desarrollaron un sentido de ubicación increíble, usted ya mira por el reojo y sabe dónde está, ya sabe que viene un andén y escribiendo ahí, entonces muchachos, eso mimo que estamos haciendo acá mire yo les estoy tratando de enseñar que hay unos tiempos para cada cosa, cuando usted está trabajando, cuando usted esté en su universidad, hay unos tiempos para cada cosa, organice su tiempo, no se vuelva dependiente de las tecnologías.

\section{De la naturaleza integradora del conocimiento profesional específico del profesorado de tecnología e informática asociado al concepto de tecnología escolar}

La naturaleza integradora del conocimiento del profesor al cual hacemos referencia debe, por lo menos, entenderse bajo tres criterios o aspectos que definen su principio integrador.

1. El primero tiene que ver con la unidad representativa de sentido del aula de clases. El concepto de tecnología como un cuerpo de conocimiento integrado se caracteriza por la naturaleza diversa y compleja que posibilita la emergencia situacional del sentido dentro del evento clase. Desde esta perspectiva, la clase es un acto de disposición y preparación colectiva para la determinación del saber. Por esta razón, la organización de este conocimiento debe reconocerse en el marco de una variedad de actos y determinaciones intencionadas que los sujetos participes de la clase aportan con el pleno de su subjetividad, para que de ello devengan actos como el de la enseñanza y del aprendizaje en función del concepto de tecnología, de tal forma que el conjunto de acciones, ya sean de carácter explícito o tácito, que hacen posible el evento denominado clase, posibiliten también la construcción de este conocimiento.

2. El segundo aspecto da cuenta de la incidencia de las fuentes de saber que nutren el conocimiento del profesor y sus respectivos lugares de producción o estatutos epistemológicos fundantes (cf. Perafán, 2011, 2015). En ese orden de ideas, se supone que el concepto de tecnología que enseña el profesor es producto de la relación histórica que este ha mantenido con el saber sobre tecnología que enseña, y dicho saber se nutre y configura a partir de cada uno de los tipos y fuentes de conocimiento constitutivas del conocimiento específico del profesor.

3. El tercer aspecto, que se articula con los dos anteriores, describe un fenómeno discursivo de producción escolar de sentido. Esta característica, 
que también explica el principio integrador del concepto de tecnología, obedece a una relación dialógica y dinámica propia de un diálogo deconstructivo de los cuatro tipos de saber visibles en la unidad de aula de clase en el dispositivo discursivo del profesor, que provoca la emergencia de una variedad de figuras literarias que proyectan las creaciones del profesorado para enseñar qué es tecnología en la escuela; hablamos, entre otros, de metáforas, símiles, imágenes, analogías, ejemplos, relatos, elementos que identifican, guían y viabilizan la enseñanza de este contenido disciplinar escolar (cf. Perafán, 2011, 2015).

Como consecuencia de las anteriores características que definen la naturaleza integradora de este conocimiento profesional específico, el concepto de tecnología escolar se puede comprender como:

\section{Una estructura relacional crítico- reflexiva que forma sujetos integrales}

El concepto de tecnología en el dispositivo discursivo del profesor es una forma de conocimiento que desde diversos posicionamientos en materia crítica y reflexiva afronta una mirada hacia la formación de un sujeto integral que actúe de manera responsable con su desarrollo y con su entorno. De acuerdo con este planteamiento, comprender la tecnología significa ser también sujetos que actuamos de manera consciente y reflexiva en cuanto a la forma de relacionarnos con todo el dispositivo tecnológico que se ha instalado en diversos escenarios de lo cotidiano, hecho que lleva a plantar el rescate de valores humanos que hacen posible nuestra convivencia con nuestros cercanos y la naturaleza que nos rodea.
Desde esta perspectiva, la dinámica del concepto de tecnología se genera a partir de: El guion de las frases asociadas a la dominación y dependencia tecnológica, la metáfora de la formación humana y la metáfora de la protección y el cuidado del medioambiente.

\section{Un dispositivo histórico de "razón polisémica del campo tecnológico"}

El concepto de tecnología es también un saber construido por este profesorado, que encierra lo que hemos denominado "razón polisémica del campo tecnológico", cuya organización por un lado comprende el despliegue de la subjetividad encaminada al descubrir, crear y proponer de los estudiantes cuando interactúan con el objeto tecnológico y, por otro lado, parte de que dicho objeto es portador de un conocimiento histórico con el cual podemos interactuar para enriquecerlo y transformarlo, más allá de su presentación como objeto material inmóvil carente de contenido abstracto. Estas características que componen la razón polisémica se refieren principalmente a lugares de enseñanza donde los estudiantes interactúan con estos objetos tecnológicos (campo tecnológico), y se estimula así su condición de sujetos aprendices en el entorno escolar, escenarios que el profesor promueve desde su orden discursivo para enseñar una noción de tecnología propia. En tal sentido, para la emergencia de la razón polisémica a la cual hemos hecho referencia, los profesores recurren al guion de la pregunta żqué es? y la metáfora de los ejemplos.

La unidad subjetiva y colectiva de creación y transformación del entorno

Esta comprensión integradora del concepto de tecnología constituye, por un lado, una acción creadora subjetiva y colectiva en materia tecnológica en la que participan estudiantes 
y profesores que repercute transformando el entorno y los modos de actuar en él, es decir, de la praxis; por otro lado, tal posicionamiento también logra encuadrarse en el escenario escolar a partir del saber histórico que se genera de este accionar creador y de la experiencia profesoral, provocando prácticas que se institucionalizan y promueven la cultura escolar. Desde esta perspectiva, el profesorado de tecnología e informática organiza su discurso de acuerdo con la metáfora del objeto, la metáfora del relato de sucesos y el ritual de proyectos y experiencias exitosas.

Un dispositivo de participación colectiva que afronta el hacer y saber hacer tecnológico en lo cotidiano

Finalmente, este concepto se traduce también como un escenario, en el cual el análisis, la discusión, la organización mental, las experiencias vivenciales, entre otros componentes, se juegan como factores determinantes que encauzan el hacer y saber hacer desde una perspectiva tecnológica contextualizada. Así, hablar de tecnología en el aula de clases implica una experiencia cercana de interacción en la cual el estudiante se enfrenta a diferentes situaciones que explotan sus capacidades en función de ser un sujeto tecnológicamente competente desde lo cotidiano. En el marco de este planteamiento, el concepto de tecnología se genera a partir del ritual de las preguntas orientadoras, el guion del reto y la metáfora del planteamiento de situaciones problema, necesidades y deseos del hombre.

\section{Conclusiones}

Para responder la pregunta de investigación que planteó este estudio: ¿̇uál es el conocimiento profesional específico construido por el profesorado de tecnología e informática asociado al concepto de tecnología escolar? Se realizó una búsqueda rigurosa y sistemática de las atribuciones de sentido que este profesorado ha construido históricamente en función de la enseñanza del concepto de tecnología de manera local y contextualizada; es decir, del saber de tecnología que se produce in situ cuando los maestros se encuentran enseñando este concepto. Así, referirnos al concepto de tecnología en este estudio pasa necesariamente por reconocerse como producto del mundo de la vida escolar y en especial de la naturaleza integradora de saberes provenientes de las prácticas de enseñanza en el aula de clases, en la cual la participación del profesor de tecnología e informática es crucial, ya que a partir de su intencionalidad de enseñar, del principio de razón formadora que constituye su subjetividad profesoral, aparece un concepto de tecnología epistemológicamente distinto que posibilita la emergencia de sujetos aprendices de este contenido de enseñanza.

En este orden de ideas, entendemos que este concepto en su acepción escolar representa una creación histórica de la propia escuela, por y para ella misma (Chervel, 1991), en el que se recupera el sentido que atribuye el profe- 
sor, como saber que educa y contribuye a la formación de sujetos. Este posicionamiento marca diferencias respecto de algunas posturas tradicionales que reconocen al saber proveniente del ámbito escolar, y en especial el que enseña el profesor, como un saber "banalizado", "vulgarizado" y además "deformado" de las disciplinas científicas, ya que su intención generatriz no es la de ampliar la aurora de conocimiento disciplinar, sino precisamente, por ser de carácter escolar, se ubica en el plano de los saberes que pretenden formar individuos bajo una disciplina escolar específica, asociada a un contenido escolar determinado, construido dentro de una comunidad de profesores.

En este sentido, el reconocimiento y estudio de este tipo de conocimiento permite revalidar la relación histórica que ha mantenido el profesor con el conocimiento con que educa. Este último ha dado lugar no solo a procesos de adaptación o recontextualización de saberes disciplinares, como se conoce tradicionalmente desde el ámbito de las didácticas específicas, sino que efectivamente existen sentidos propios producidos por los profesores que provocan la emergencia de sujetos aprendices de diversas categorías escolares de enseñanza. Desde esta perspectiva, se resalta el dispositivo discursivo del profesorado como un escenario dinámico y potente que encierra en su estructura interna una articulada red de significados y sentidos que operan bajo el acto intencionado de enseñanza, la cual se hace necesario seguir estudiando en profundidad.

Así, la integración de los sentidos atribuidos por el profesorado de tecnología e informática sobre el concepto de tecnología, construidos desde el aula de clases como unidad representativa de significado, las fuentes de saber y sus respectivos estatutos epistemológicos, así como también del fenómeno discursivo de producción escolar de sentido, permite comprender este concepto como:

- Una estructura relacional crítico-reflexiva que forma sujetos integrales para actuar de manera consciente, crítica y reflexiva, que media la forma de relacionarnos con todo el dispositivo tecnológico que se ha instalado en diversos escenarios de lo cotidiano, y plantea el rescate de valores humanos que hacen posible la convivencia con nuestros cercanos y el entorno, de una forma más amigable.

- Un dispositivo histórico de "razón polisémica del campo tecnológico", que comprende, por un lado el despliegue de la subjetividad de los estudiantes encaminada al descubrir, crear y proponer cuando interactúan con el objeto tecnológico, y, por otro lado, determinar que dicho objeto porta un conocimiento histórico con el cual es posible interactuar para enriquecerlo y transformarlo, más allá de su consideración como objeto material inmóvil carente de contenido abstracto.

- La unidad subjetiva y colectiva de creación y transformación del entorno, donde participan estudiantes y profesores, que inciden por un lado en la praxis cotidiana y, por otro lado, en la institucionalización de prácticas tecnológicas que promueven la cultura escolar.

- Un dispositivo de participación colectiva que afronta el hacer y saber hacer tecnológico en lo cotidiano, en el cual el análisis, la discusión, la organización mental y las experiencias vivenciales, entre otros componentes, se juegan como factores determinantes de este hacer y saber hacer, 
para permitir con ello una experiencia cercana de interacción, en la cual el estudiante se enfrenta a diferentes situaciones que explotan sus capacidades en función de ser un sujeto tecnológicamente competente desde su contexto.

La identificación de este concepto de tecnología propio de este profesorado lleva a plantearse cuestionamientos respecto de cómo se aborda el trabajo del docente, su papel en las prácticas de enseñanza y en especial sobre su reconocimiento como intelectual productor de sentido y trabajador de la cultura. Tradicionalmente la comprensión sobre su accionar en el aula con los objetos de enseñanza y su contribución a la aparición de los mismos pareciese carecer de significado, quizás estar expuesta a un solo tipo de comprensión que supone de manera privilegiada procesos de modelación, transformación y recontextualización. No obstante, nuestro planteamiento circula más en función del sentido que construye el profesorado como unidad subjetiva cargada de significado histórico, que ha posibilitado de manera particular la enseñanza de contenidos escolares en función de educar sujetos, y que -como hemos podido apreciar a lo largo de esta investigación- el concepto de tecnología escolar construido por el profesorado de tecnología e informática se presenta como una evidencia empírica que aporta a la validación y consolidación de esta perspectiva.

Insistimos en que no se trata de reivindicar el carácter disciplinar del conocimiento del profesor de manera exclusiva bajo la aurora de un saber disciplinar específico de referencia o de condición externa. La intención se centra en el posicionamiento del conjunto de atribuciones de significado que hacen los profesores cuando enseñan las nociones y los conceptos pertenecientes a las disciplinas que circulan en el entorno escolar. Ello sería una consideración para la apertura del carácter disciplinar propio de la profesión docente, que soporta al mismo tiempo características epistemológicas propias del conocimiento profesional del profesor.

\section{Referencias bibliográficas}

Barinas, G. (2014). Tecné, Episteme y Didaxis: TED. [Número extraordinario]. Memorias Sexto Congreso Internacional sobre Formación de Profesores de Ciencias. 8 al 10 de octubre. Bogotá.

Barinas, G. y Perafán, G. A. (2014 enero-junio). El conocimiento profesional específico del profesor de ciencias asociado a una categoría de enseñanza: estudio de caso sobre la noción de célula. EDUCYT, 10 (ISSN 2215-8227).

Bolívar, A. (2005). Conocimiento didáctico del contenido y didácticas específicas. Profesorado: Revista de Currículum y Formación del Profesorado, 2(9), 1-39.

Bromme, R. (1988). Conocimientos profesionales de los profesores. Enseñanza de las Ciencias, 6(1), 19-29. 
Carr, W. y S. Kemmis (1988). Teoría crítica de la enseñanza. La investigación en la acción en la formación del profesorado. Barcelona: Martínez Roca.

Chervel, A. (1991). Historia de las disciplinas escolares. Reflexiones sobre un campo de investigación. Revista de Educación, 295, 7-37.

Creswell, J. (1998). Qualitative inquiry and research design. Choosing among five traditions. USA: Sage.

Furió, C. (1994). Tendencias actuales en la formación del profesorado de Ciencias. Enseñanza de las Ciencias, 2(2), 188-199.

García, M. (1997). Conocimiento profesional del profesor de matemáticas. El concepto de función como objeto de enseñanza-aprendizaje. Sevilla: Universidad de Sevilla.

Gil, D. (1991). ¿Qué hemos de saber y saber hacer los profesores de ciencias? Enseñanza de las Ciencias, 9(1), 69-77.

Gimeno, J. y Pérez, A. I. (1988). Pensamiento y acción en el profesor: de los estudios sobre la planificación al pensamiento práctico. Infancia y Aprendizaje, 42, 37-63.

Hashweh, M. Z. (2005). Teacher pedagogical constructions: A reconfiguration of pedagogical content knowledge. Teachers and Teaching, 17 (3), 273-292.

Hernández, S.; Fernández, C. y Baptista, P. (2008). Metodología de la investigación (4. ${ }^{a}$ ed.). México: McGraw-Hill Interamericana.

Jackson, Ph. W. (1968). La vida en las aulas. Madrid: Morata.

Janesick, V. (1998). Stretching: Exercises for qualitative researcher. Thousand Oaks, CA, EE. UU.: Sage.

Marcelo, G. C. (1993). Cómo conocen los profesores la materia que enseñan. Algunas contribuciones de la investigación sobre conocimiento didáctico del contenido. En L. Montero, y J. Vez, (eds.). Las didácticas específicas en la formación del profesorado (pp. 151-185). Santiago de Compostela: Tórculo.

Martínez, C. (2000). Las propuestas curriculares sobre el conocimiento escolar en el área de conocimiento del medio: dos estudios de caso en profesores de primaria (tesis doctoral). Universidad de Sevilla, España.

McCormick, R, (2008). Classroom case studies. En H. E. Middleton (ed.) Researching Technology Education (pp. 6-27). Rotterdam: The Netherlands: Sense Publishers.

Merriam, S. (1998). Qualitative research and case study applications in education. San Francisco, California: Jossey-Bass.

Middleton, H. E. (2008). Knowledge researching technology education. Rotterdam, The Netherlands: Sense Publishers.

Park, S. \& Oliver, J. S. (2008). Revisiting the conceptualisation of pedagogical content knowledge (PCK): $\mathrm{PCK}$ as a conceptual tool to understand teachers as professionals. 
Research in Science Education, 38(3), 261-284. doi: http://doi.org/10.1007/ s1 $1165-007-9049-6$.

Perafán, G. (2004). Epistemología del profesor sobre su propio conocimiento profesional. Bogotá: Universidad Pedagógica Nacional.

Perafán, G. A. (201 1). Conocimiento profesional docente: nuevas perspectivas epistemológicas y metodológicas. Instrumentos de investigación: Analytical Scheme. Proyecto de investigación Universidad Pedagógica Nacional.

Perafán, G. A. (2012, octubre 3-6). La transposición didáctica como estatuto epistemológico fundante de los saberes académicos del profesorado de ciencias (III). Ponencia presentada en la Segunda Conferencia Latinoamericana del International History, Philosophy, and Science Teaching Group. Mendoza: Argentina.

Perafán, G. (2013a). La transposición didáctica como estatuto epistemológico fundante de los saberes académicos del profesor (I). Folios, 37, 83-93.

Perafán, G. A. (2013b). El conocimiento profesional docente: caracterización, aspectos metodológicos y desarrollo. Estado de la enseñanza de las ciencias: 2000-201 1. Ministerio de Educación Nacional-Universidad del Valle.

Perafán, G. A. (2015). Conocimiento profesional docente y prácticas pedagógicas. El profesorado como productor de conocimiento disciplinar-profesional. Bogotá: Aula de Humanidades.

Ponte, J. (2012). Estudiando el conocimiento y el desarrollo profesional del profesorado de matemáticas. En N. Planas (comp.). Teoría, crítica y práctica de la educación matemática. Barcelona: Grao.

Porlán, A. R., y Rivero, G. A. (1998). El conocimiento de los profesores. Sevilla: Diada.

Rodríguez, G.; Gil, J. y García, E. (1999). Metodología de la investigación cualitativa. Málaga: Aljibe.

Shulman, L. S. (1986). Those who understand: Knowledge growth in teaching. Educational Researcher, 15(2), 4-14.

Shulman, L. S. (1987). Knowledge and teaching: foundations of new reform. Harvard Educational Review, 57(1), 1-22 [Ed. en español: Conocimiento y enseñanza: fundamentos de la nueva reforma. Profesorado. Revista de Currículum y Formación del Profesorado, 9(2), 30. 2005].

Stake, R. (1995). Investigación con estudio de casos. Madrid: Morata.

Stake, R. (2005). Qualitative case studies. En N. Denzin y Y. Lincoln (2005). The Sage handbook of qualitative research (3. ${ }^{a}$ ed.). Londres: Sage Publications.

Wolcott, H. (1990). Writing up qualitative research. California: Sage Publications.

Yin, R. K. (2003). Case study research: Design and methods. Thousand Oaks: Sage. 